Supplementary Information for

\title{
Structure and Mechanical Stabilities of the Three-Way Junction Motifs in Prohead RNA
}

Boyang Zhuo ${ }^{1}$, Xinwen $\mathrm{Ou}^{1}$, Jingyuan $\mathrm{Li}^{1 *}$

${ }^{1}$ Department of Physics, Zhejiang University, Hangzhou 310027, China

Email: jingyuanli@zju.edu.cn (J.L.) 


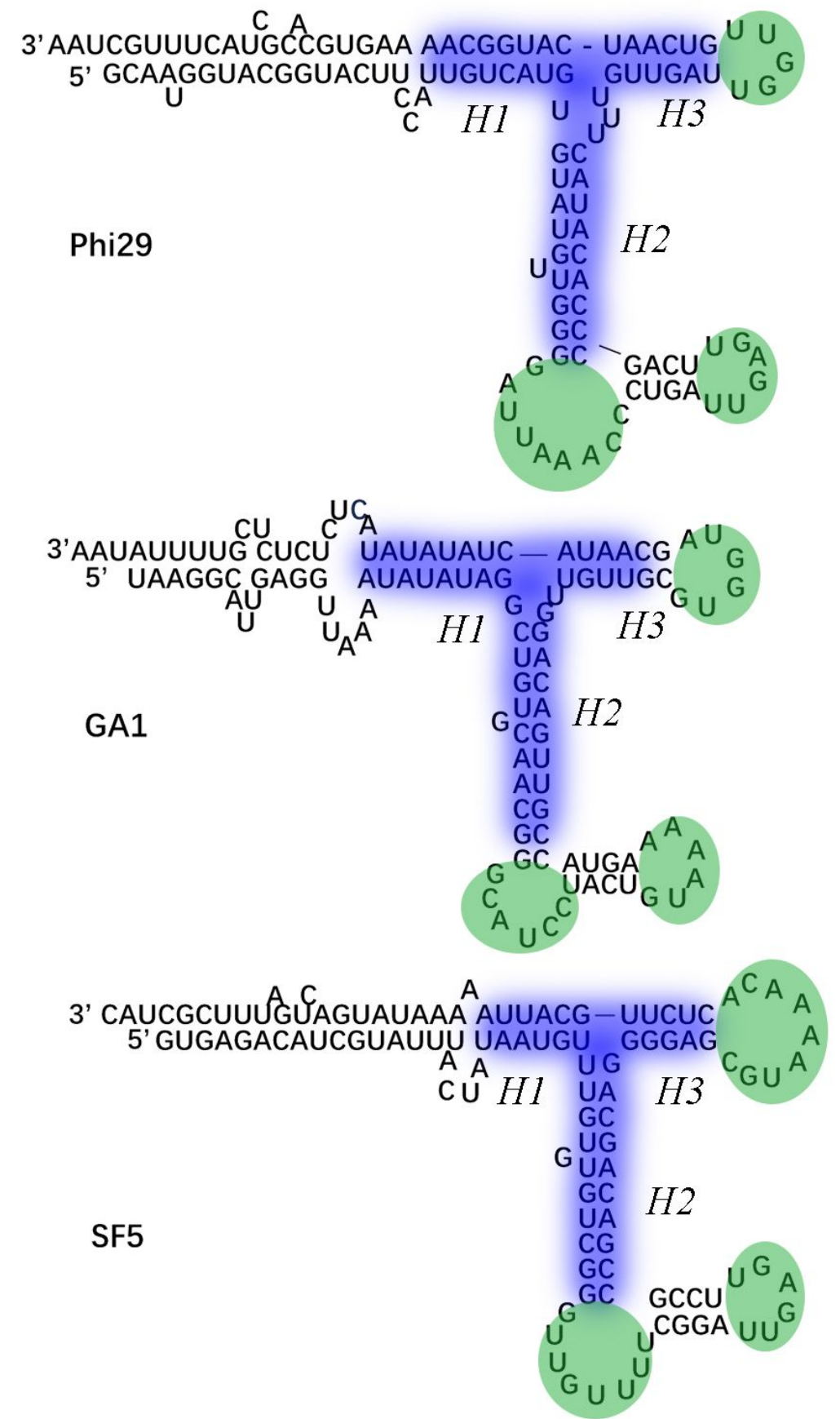

Figure S1. Secondary structures and sequences of wild-type pRNAs. The three singlebase bulges are highlighted in green and the $3 \mathrm{WJ}$ in blue. 


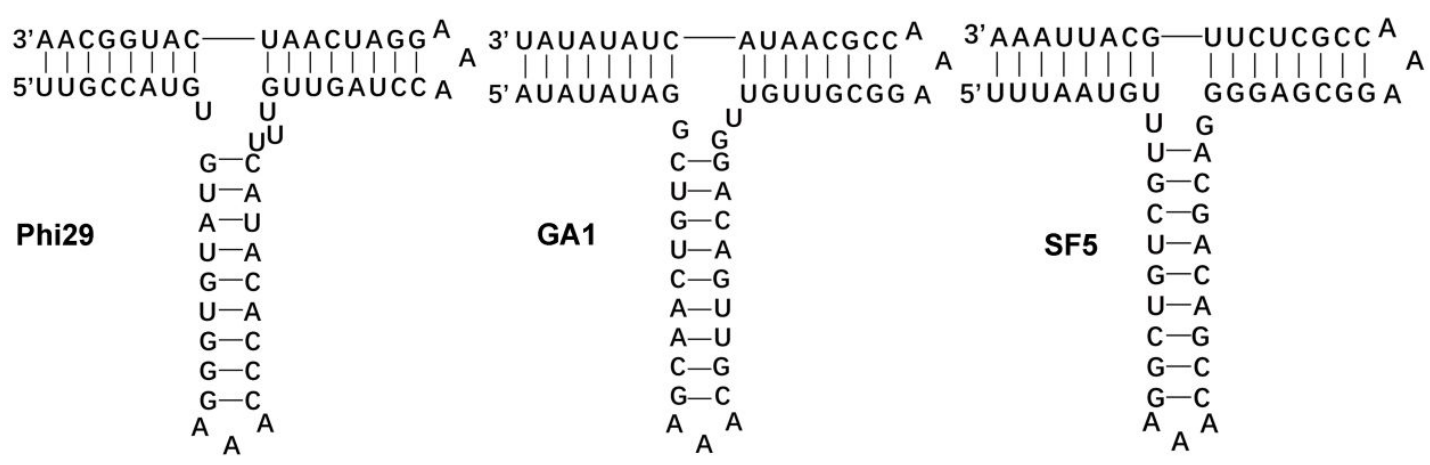

Figure S2. The sequences of phi29, GA1, and SF5 3WJ-pRNAs with apical loop since RNAcomposer is designed to predict the tertiary structure for one RNA strand.

(a)

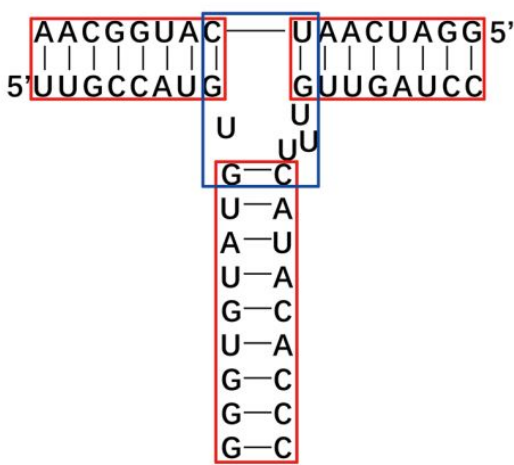

(c)

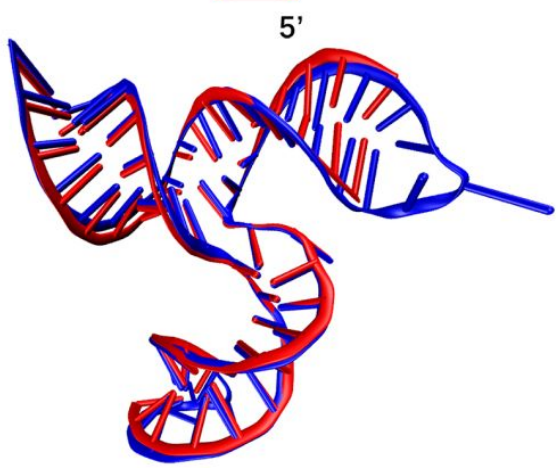

(b)

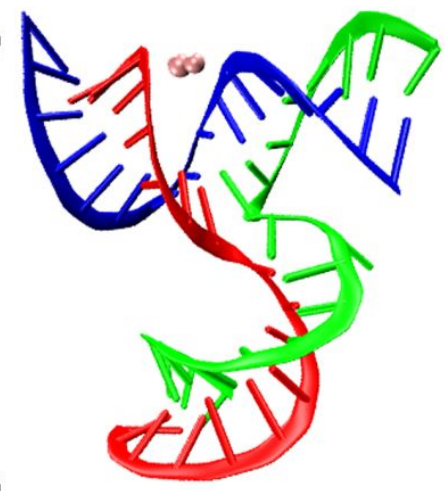

(d)

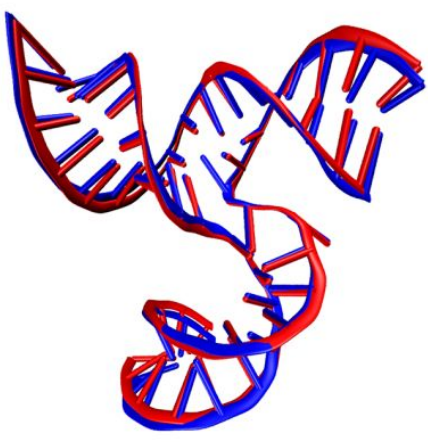

Figure S3. (a and b) Schematic of phi29 3WJ-pRNA in the crystal structure and the tertiary structure of phi29 3WJ-pRNA from Protein Data Bank, PDB: 4kz2. The tertiary structure of phi29 3WJ-pRNA is divided into three stems (red box) and one loop (blue box) as templates. (c and d) Comparison of the predicted phi29 3WJpRNA structure (blue) by RNAcomposer and the crystal structure (red). The heavyatom RMSD of the predicted structure with respect to the crystal structure is $\sim 0.97 \AA$. It suggests the predicted structure is consistent with the experiment result, and the influence of added apical loops is negligible. 

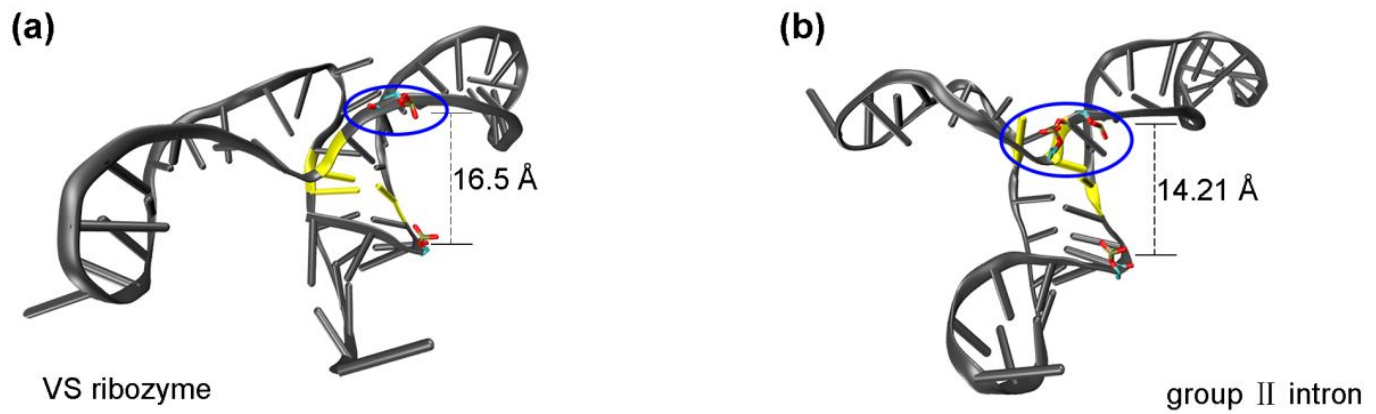

Figure S4. (a) The structure of VS ribozyme (PDB: 2MTK). The unpaired bases in the junction of the coaxial helical stack are indicated in yellow. The phosphate groups in the junction migrate outside the major groove (blue circle) and increase the major groove width (i.e. 15.14 - $16.51 \AA$ ). (b) The structure of group II intron (PDB: 2LU0). Bases engaged in A-minor tertiary interactions (highlighted in yellow) cause the phosphates of the bases to point outside at the junction, resulting in the increase of the major groove width (i.e. 13.67 - $15.32 \AA$ ).

(a)

(c)
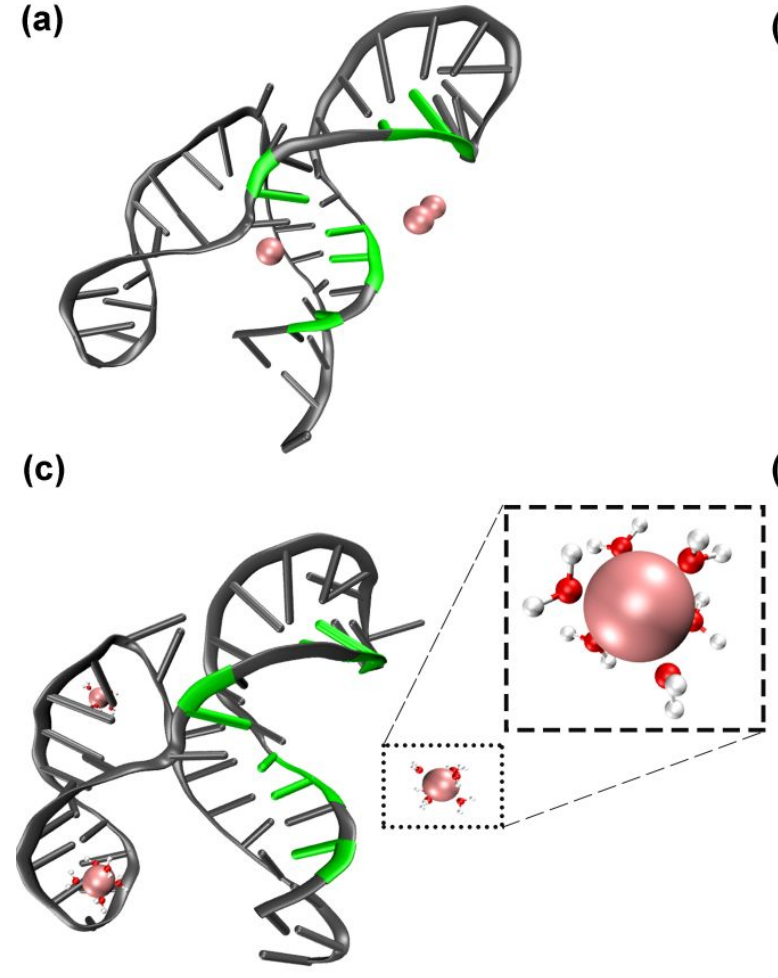

(b)

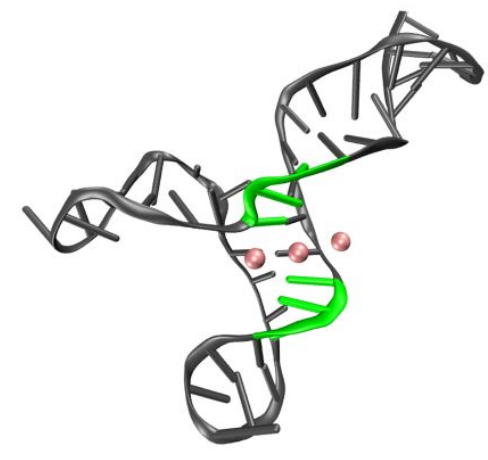

(d)

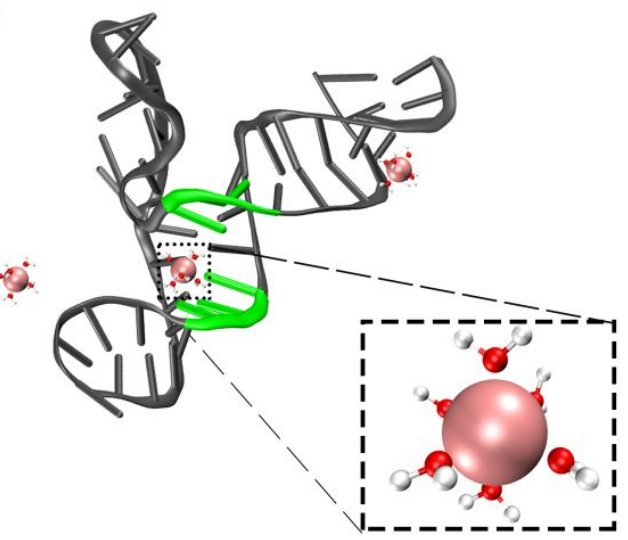

Figure S5. The structures of VS ribozyme and group $I$ intron with three introduced $\mathrm{Mg}^{2+}$ (a and $\mathbf{b}$ ) The three closest base pairs inside the major groove between coaxial helices of $3 \mathrm{WJ}$ are colored in green. The three introduced $\mathrm{Mg}^{2+}$ ions are colored in pink. (c and d) The structures of VS ribozyme and group II intron after 10 ns relaxation simulation. The three introduced $\mathrm{Mg}^{2+}$ ions diffuse away from the initial sites. Inset, these $\mathrm{Mg}^{2+}$ ions become fully hydrated. 

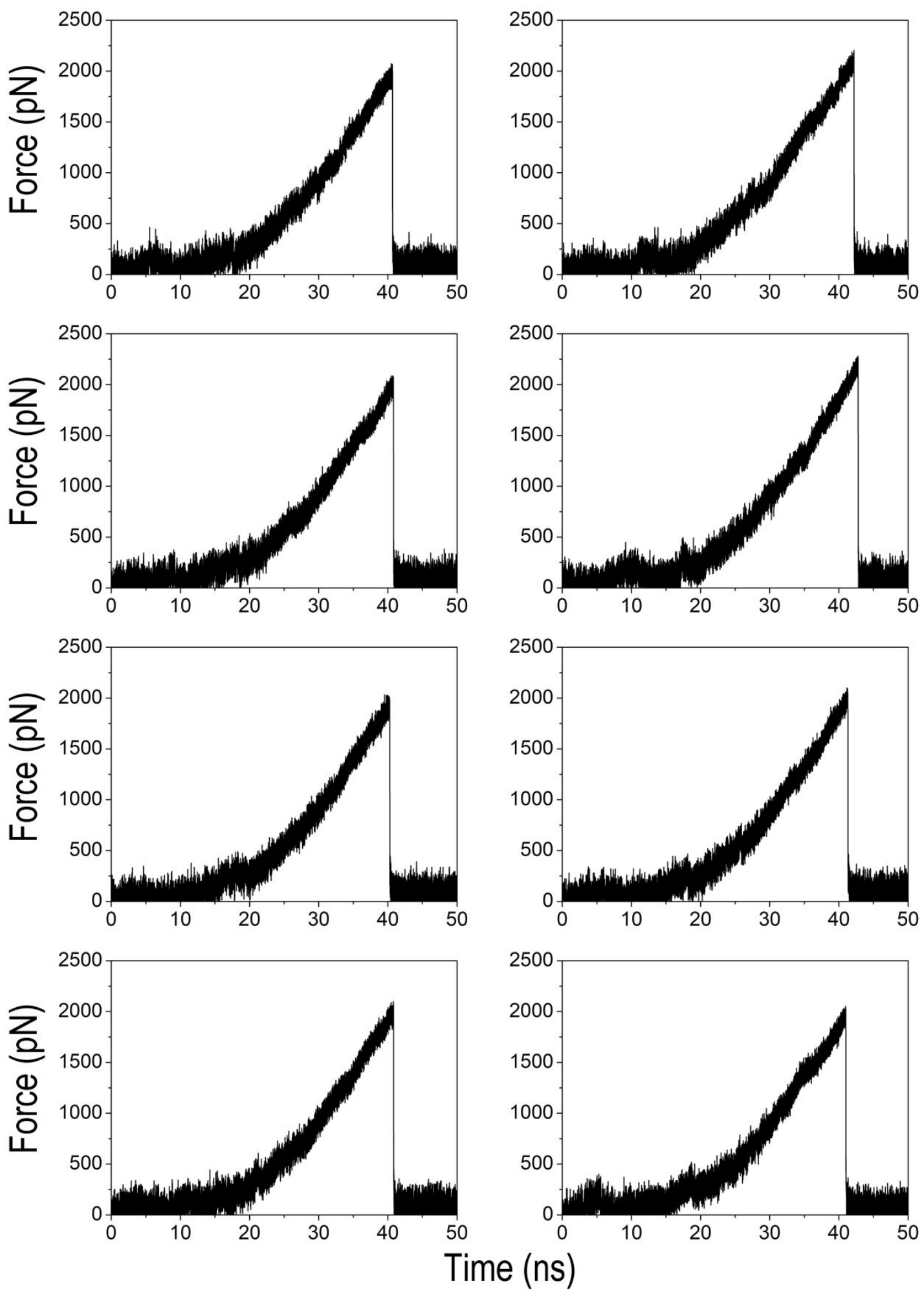

Figure S6. Force profiles of GA1 3WJ-pRNA during the unfolding along H1-H3 (eight trajectories). The rupture forces are 2027, 2206, 2087, 2281, 2033, 2101, 2099, and $2051 \mathrm{pN}$; the average rupture force is $2111 \pm 83 \mathrm{pN}$. 

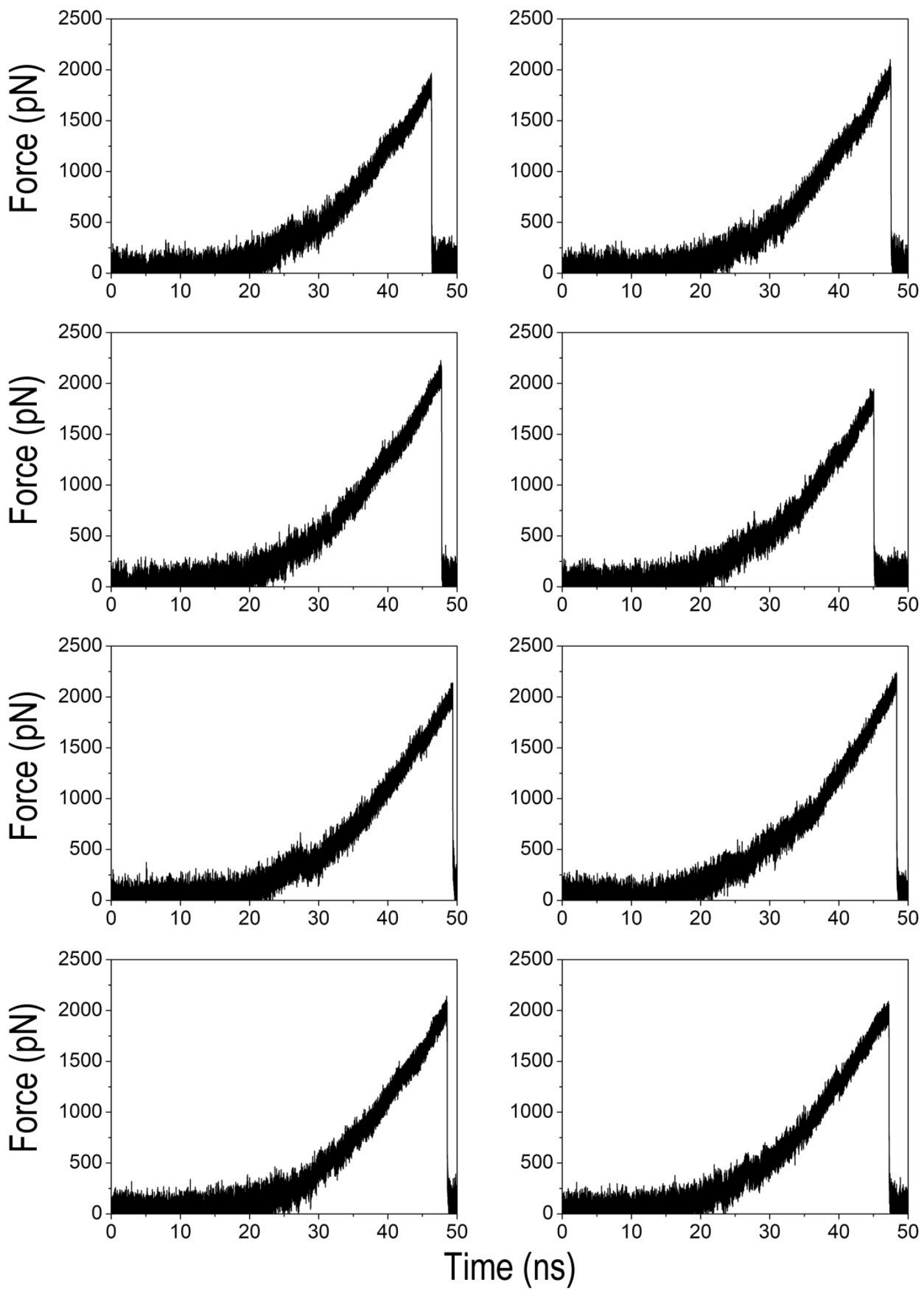

Figure S7. Force profiles of SF5 3WJ-pRNA during the unfolding along H1-H3 (eight trajectories). The rupture forces are 1969, 2102, 2225, 1946, 2140, 2242, 2141, and $2091 \mathrm{pN}$; the average rupture force is $2107 \pm 100 \mathrm{pN}$. 


\section{VS ribozyme and group II intron MD simulation}

The structures of VS ribozyme (PDB: 2MTK) and group II intron (PDB: 2LU0) were obtained from the protein data bank. Three $\mathrm{Mg}^{2+}$ ions were introduced into three closest base pairs inside the major groove of both RNAs [A3 - U22, C5 - C19, and U6 - A18 for VS ribozyme; C9 - G36, U10 - U35, and C11 - G33 for group II intron]. Both 3WJ RNA configurations were solvated with the TIP3P water, and $\mathrm{Na}^{+}$and $\mathrm{Cl}^{-}$ ions were added to neutralize the system and adjust the total ionic strength to $100 \mathrm{mM}$. All ions were placed randomly in the solvent box. The simulation system of VS ribozyme contained $\sim 76000$ atoms with a box size of $8 * 12 * 8 \mathrm{~nm}^{3}$, and the simulation system of group II intron contained $\sim 59000$ atoms with a box size of $10 * 6 * 10 \mathrm{~nm}^{3}$. The solvated systems were first energy-minimized with 10000 steps, and 5 ns NPT equilibration runs were then conducted with harmonic restraints on RNA heavy atoms and $\mathrm{Mg}^{2+}$ ions $(P=1 \mathrm{~atm}, T=300 \mathrm{~K})$. Subsequently, the RNA conformations were further relaxed for $5 \mathrm{~ns}$ NPT simulation by only restraining RNA heavy atoms and the final conformations were adopted for the following 10-ns production simulation.

The AMBER ff 99 force field with parmbsc 0 and $\chi_{\mathrm{OL} 3}$ was used for all nucleotides, and all-atom MD simulations were carried out using the GROMACS software package version 5.1. The $\mathrm{Mg}^{2+}$ ion model developed by Allner's work was used in all simulation runs. The Joung-Cheatham parameters were used for $\mathrm{Na}^{+}$and $\mathrm{Cl}^{-}$ions. The NoséHoover thermostat and the Parrinello-Rahman barostat were chosen for temperature and pressure control, respectively. The short-range electrostatic and van der Waals interactions were calculated at a cutoff distance of $12 \AA$, whereas the long-range electrostatic interactions were treated using the particle mesh Ewald method. The SHAKE algorithm was used to constrain all bonds involving hydrogen. Periodic boundary conditions were applied in all directions, and an integration step of 2 fs was used for all simulations. Simulation snapshots and analysis of $3 \mathrm{WJ}$ RNA were performed in visual molecular dynamics. 